\title{
Remainders in pointfree topology
}

\author{
Maria João Ferreira ${ }^{\mathrm{a}}$, Jorge Picado ${ }^{\mathrm{a}, *}$, Sandra Marques Pinto ${ }^{\mathrm{a}}$ \\ ${ }^{a}$ CMUC, Department of Mathematics, University of Coimbra, 3001-501 Coimbra, Portugal
}

\begin{abstract}
Remainders of subspaces are important e.g. in the realm of compactifications. Their extension to pointfree topology faces a difficulty: sublocale lattices are more complicated than their topological counterparts (complete atomic Boolean algebras). Nevertheless, the co-Heyting structure of sublocale lattices is enough to provide a counterpart to subspace remainders: the sublocale supplements. In this paper we give an account of their fundamental properties, emphasizing their similarities and differences with classical remainders, and provide several examples and applications to illustrate their scope. In particular, we study their behaviour under image and preimage maps, as well as their preservation by pointfree continuous maps (i.e. localic maps). We then use them to characterize nearly realcompact and nearly pseudocompact frames. In addition, we introduce and study hyper-real localic maps.
\end{abstract}

Keywords: Frame, locale, sublocale, Heyting algebra, coframe, pseudodifference, supplement, remainder, localic map, remainder preservation, proper map, Stone-Čech compactification, regular Lindelöf reflection, realcompact reflection, nearly realcompact, nearly pseudocompact, hyper-real map.

2010 MSC: 06D22, 06D20, 54D40, 54C10.

\section{Introduction}

In general topology, by a remainder of a Tychonoff space $X$ it is usually understood the subspace $b X \backslash X$ of some compactification $b X$ of $X$. Remainders of subspaces and their preservation by continuous maps play an important role in some classical results. E.g., by the Henriksen-Isbell Theorem (cf. [21]), a continuous map $f: X \rightarrow Y$ of Tychonoff spaces is proper (= perfect $[19,3.7])$ if and only if any of the following equivalent conditions hold:

(R1) The Stone-Čech extension $\beta(f): \beta X \rightarrow \beta Y$ of $f$ takes remainder to remainder, that is,

$$
\beta(f)[\beta X \backslash X] \subseteq \beta Y \backslash Y .
$$

\footnotetext{
* Corresponding author

Email addresses: mjrf@mat.uc.pt (Maria João Ferreira), picado@mat.uc.pt (Jorge Picado), sandra@mat.uc.pt (Sandra Marques Pinto)
} 
(R2) For every compactification $\kappa Y$ of $Y$, the extension $\tilde{f}: \beta X \rightarrow \kappa Y$ of $f$ takes remainder to remainder, that is,

$$
\tilde{f}[\beta X \backslash X] \subseteq \kappa Y \backslash Y .
$$

Hence, in the point-set context, remainder preserving maps are precisely the proper maps.

This provides nice categorical characterizations of proper maps since remainder preserving condition (R1) means precisely that the square

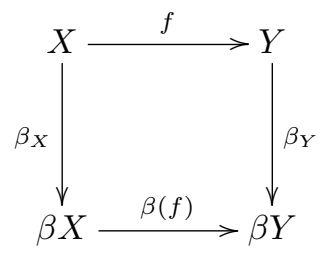

is a pullback diagram (i.e., $f$ is $\beta$-cartesian [35]), while (R2) is equivalent to the fact that

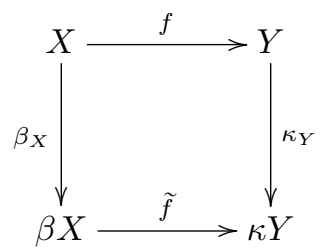

is a pullback diagram. (For a broad categorical approach to properness and perfectness see [14] and [35].)

The generalization of Henriksen-Isbell Theorem to pointfree topology faces a difficulty: unlike the algebra $\mathcal{P}(X)$ of subspaces of a space $X$, the sublocale lattice $\mathcal{S}(L)$ of a locale (frame) $L$ is generally not Boolean, and therefore complements (and hence the difference of two sublocales) do not necessarily exist. He and Luo [20] circumvented this by grabbing the categorical conditions rather than (R1) and (R2) to characterize proper maps of locales:

Theorem 1.1. [20, Theorem 1] Let $f: L \rightarrow M$ be a localic map between completely regular locales. Then the following statements are equivalent:

(i) $f$ is proper.

(ii) For the Stone-Čech compactification $\beta_{M}: M \rightarrow \beta M$ of $M$, the following diagram is a pullback square:

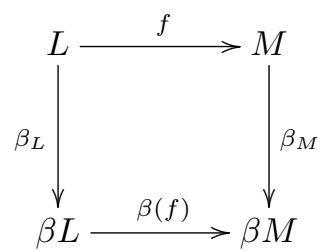


(iii) For every compactification $\kappa_{M}: M \rightarrow \kappa M$ of $M$, the following diagram is a pullback square:

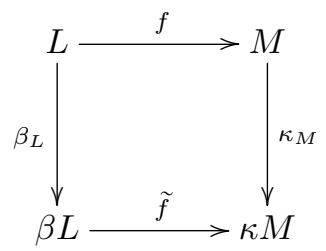

Nevertheless, in spite of being no longer a (atomic) Boolean algebra, $\mathcal{S}(L)$ is always a coframe (i.e., the dual of a frame). This means that $\mathcal{S}(L)$ is a co-Heyting algebra and, therefore, 'residuated' in the sense that there is a binary operation that acts like a subtraction. The existence of such an operation permits the computation of residuals $L \backslash S$ in the absence of a unary complement operation.

Thus, the class of localic maps that take remainder to remainder i.e., that satisfy the counterpart of conditions (R1) or (R2) with respect to that subtraction, still remains to be studied, and compared with that of proper maps.

In [17], Dube and Naidoo approached remainder preservation with a definition heavily dependent on the point spectra of the generalized pointfree spaces. It is the aim of this paper to make the notion of remainders and remainder preservation truly pointfree, by investigating the natural, alternative, direct approach based on the co-Heyting structure of sublocale lattices, with no reference to points whatsoever. We would like to stress that this approach is not new in the literature. In fact, one may find it, formulated in terms of the frame of congruences, somewhat hidden amidst the study of some perfect compactifications in a paper by D. Baboolal ([1]; see also [2] where it appears in an even more particular situation). Our goal here is to collect the basic algebraic structure and results for its use in the general pointfree setting.

Note that the fundamental fact that, for any frame $L, \mathcal{S}(L)$ is generally a coframe rather than a frame is, after all, a pleasant surprise. Actually, in the classical case, the Heyting operator $A \rightarrow B$ in the Boolean $\mathcal{P}(X)$ is given by $\neg A \cup B$, never used in pointset topology, whereas it is the co-Heyting operator given by the set-theoretic difference $B \cap \neg A=B \backslash A$ that is actually used. Thus, in the category of locales one should take the co-Heyting operator in $\mathcal{S}(L)$ as the natural substitute for the set-theoretic difference. This idea goes back to Isbell and Plewe [23, 32, 33] (cf. [29, VI.5]) and provides the right definition for the remainder of a locale and the corresponding concept of mapping remainder preservation.

The paper is organized as follows. In the first sections, we mostly survey familiar material of pointfree topology and lattice theory (but also including a few new results) that are of relevance for the study of remainders. More specifically, we start in Section 2 with some basic background on the categories of frames and locales, and in Section 3 we survey the structure of coframes (meaning complete co-Heyting algebras, the dual lattices of frames) and the properties of the co-Heyting operator (here called pseudodifference operator). Then, in Section 4, we look for additional properties of pseudodifferences in the more special coframe of sublocales of a locale. Section 5 deals with their behaviour under image and preimage maps. Remainders and remainder preservation are introduced and studied respectively in sections 6 and 7 and in Section 8 we compare remainder 
preserving maps to proper maps. In particular, Section 6 provides several examples that illustrate the usefulness of our approach (namely, in the study of the Alexandroff, StoneCech and Freudenthal compactifications). In Section 9, we present some more illustrative examples, now concerning the study of some particular classes of locales and localic maps as e.g. nearly realcompact and nearly pseudocompact locales and hyper-real localic maps. Finally, in the last section we compare our approach to the previous treatment by Dube and Naidoo [18].

Some of the results in this paper were presented for the first time by J. Picado at the conference held at the University of Cape Town in March 2016 to celebrate Bernhard Banaschewski's 90th birthday. We were pleased to see in a very recent paper ([16]) that T. Dube uses our approach to characterize realcompact locales.

\section{Frames and locales}

In the pointfree (localic) approach to topology, topological spaces are replaced by locales, seen as generalised spaces where points are not explicitly mentioned. Formally, a locale $L$ is defined to be a special complete lattice (where we denote top, resp. bottom, by 1 , resp. 0), usually called a frame, in which finite meets distribute over arbitrary joins, i.e.

$$
a \wedge \bigvee S=\bigvee\{a \wedge b \mid b \in S\} \quad \text { for all } a \in L \text { and } S \subseteq L
$$

Thus, in a frame $L$ the mappings $(x \mapsto(a \wedge x)): L \rightarrow L$ preserve suprema and hence we have the right Galois adjoints $(y \mapsto(a \rightarrow y)): L \rightarrow L$, satisfying

$$
a \wedge x \leqslant y \quad \text { iff } \quad x \leqslant a \rightarrow y
$$

and making $L$ a (complete) Heyting algebra. The element $a \rightarrow y$ (the relative pseudocomplement of $a$ with respect to $y$ ) is given by the formula

$$
a \rightarrow y=\bigvee\{x \mid a \wedge x \leqslant y\} .
$$

The (absolute) pseudocomplement of $a$ is the element

$$
a^{*}=a \rightarrow 0=\bigvee\{x \mid x \wedge a=0\} .
$$

If $X$ is a topological space we have the frame $\mathfrak{O} X$ of its open sets. A frame is spatial if it is isomorphic with some $\mathfrak{O} X$.

Regarding morphisms, the role of the usual continuous functions is taken by those maps $f: L \rightarrow M$ between locales, called localic maps [29], such that, for every $a \in L$, $b \in M, S \subseteq L$,

(L1) $f(\bigwedge S)=\bigwedge f[S]$ (and, in particular, $f(1)=1)$,

(L2) $f\left(f^{*}(b) \rightarrow a\right)=b \rightarrow f(a)$, and

(L3) $f(a)=1 \Rightarrow a=1$, 
where $f^{*}: M \rightarrow L$ denotes the left adjoint of $f$ provided by property (L1). These left adjoints are the frame homomorphisms, i.e. the maps between frames that preserve arbitrary joins (in particular, the top element 1) and finite meets (in particular, the bottom element 0). Note that, for each frame homomorphism $h: M \rightarrow L, h\left(x^{*}\right) \leqslant h(x)^{*}$ for every $x \in M$.

If $f: X \rightarrow Y$ is a continuous maps of spaces, we have the frame homomorphism $\mathfrak{O}(f): \mathfrak{O} Y \rightarrow \mathfrak{O} X$ defined by $\mathfrak{O}(f)(V)=f^{-1}[V]$ for every $V \in \mathfrak{O} Y$.

Locales and localic maps form the category Loc of locales while frames (= locales) and frame homomorphisms form precisely its dual category Frm. Our references for locales and frames are [25] and [29]. Here we just recall the definitions of some of the main classes of frames mentioned along the paper.

A frame $L$ is compact (resp. Lindelöf) whenever $\bigvee A=1$ for $A \subseteq L$ implies $1=\bigvee B$ for some finite (resp. countable) $B \subseteq A$. A frame $L$ is regular if, for each $a \in L$, $a=\bigvee\{b \in L \mid b<a\}$ where $b<a$ (' $b$ is rather below $a^{\prime}$ ) means that $b^{*} \vee a=1$. The completely below relation $<<$ is the interpolative modification of the rather below relation. Elements $a, b \in L$ satisfy $b \prec<a$ if and only if there exists a subset $\left\{a_{q} \mid q \in\right.$ $[0,1] \cap \mathbb{Q}\} \subseteq L$ with $a_{0}=b$ and $a_{1}=a$ such that $a_{p}<a_{q}$ whenever $p<q$ in $[0,1] \cap \mathbb{Q}$. A frame $L$ is completely regular if, for each $a \in L, a=\bigvee\{b \in L \mid b<<a\}$.

The points of a locale $L$ are the prime (or meet-irreducible) elements, that is, the $p \in L \backslash\{1\}$ such that $p=a \wedge b$ implies $p=a$ or $p=b$. A special kind of points are the completely prime elements of $L$ that satisfy the condition $p=\bigwedge S \Rightarrow p \in S$ for every $S \subseteq L$. For any locale $L$, its spectrum $\Sigma L$ is the space of all points of $L$ with the open sets $\Sigma_{a}=\{p \mid a \$ p\}, a \in L$.

\section{Coframes}

Dual lattices of frames, that is, complete co-Heyting algebras play a crucial role in this paper. As usual, we refer to them as coframes. Thus, in a coframe $L$ the mappings $(y \mapsto(a \vee y)): L \rightarrow L$ have left Galois adjoints $(x \mapsto(x \backslash a)): L \rightarrow L$, satisfying

$$
x \backslash a \leqslant y \quad \text { iff } \quad x \leqslant a \vee y .
$$

Hence we have an extra operation, the co-Heyting operation $x \backslash a$ given by the formula

$$
x \backslash a=\bigwedge\{y \mid x \leqslant a \vee y\} .
$$

We refer to $x \backslash a$ as the relative pseudodifference of $a$ with respect to $x$.

Clearly, $(-) \backslash a$ being a left adjoint, we have

$$
\left(\bigvee_{i \in J} b_{i}\right) \backslash a=\bigvee_{i \in J}\left(b_{i} \backslash a\right)
$$

From (3.1) we can also obtain the contravariant adjunction

$$
c \backslash a \leqslant b \quad \text { iff } \quad c \backslash b \leqslant a
$$

which yields

$$
b \backslash \bigwedge_{i \in J} a_{i}=\bigvee_{i \in J}\left(b \backslash a_{i}\right)
$$


Each Boolean algebra is both a Heyting and a co-Heyting algebra: just set

$$
a \rightarrow b=\neg a \vee b \quad \text { and } \quad b \backslash a=b \wedge \neg a .
$$

Dualising the proofs in [29, Prop. III.3.1.1], one gets immediately the following:

Proposition 3.1. In any complete co-Heyting algebra L we have:

(P1) $a \backslash a=0$ and $a \backslash 0=a$ for all $a$.

(P2) $b \leqslant a \quad$ iff $\quad b \backslash a=0$

(P3) $b \backslash a \leqslant b$.

(P4) $b \backslash a=(b \vee a) \backslash a$.

(P5) $a \vee(b \backslash a)=a \vee b$.

(P6) $a \backslash(a \backslash b) \leqslant a \wedge b$.

(P7) $a \vee b=a \vee c \quad$ iff $\quad b \backslash a=c \backslash a$.

(P8) $c \backslash(a \vee b)=(c \backslash b) \backslash a$ and therefore $(c \backslash b) \backslash a=(c \backslash a) \backslash b$.

(P9) for every $a, b \in L, \quad b=(b \wedge a) \vee(b \backslash a)$.

The (absolute) pseudodifference (supplement in $[26,32]$ ) of an element $a$ is the element

$$
a^{\prime}=1 \backslash a=\bigwedge\{y \mid a \vee y=1\} .
$$

Of course, $a \vee a^{\prime}=1$ (and $a^{\prime}$ is the smallest $x$ such that $a \vee x=1$ ) but in general $a \wedge a^{\prime} \geqslant 0$. The following properties are also obvious:

Proposition 3.2. In any complete co-Heyting algebra L we have:

(1) $a \leqslant b \Rightarrow a^{\prime} \geqslant b^{\prime}$.

(2) $a^{\prime \prime} \leqslant a$ and $a^{\prime \prime \prime}=a^{\prime}$.

(3) $0^{\prime}=1$ and $1^{\prime}=0$.

(4) $a^{\prime}=0$ iff $a=1$.

(5) $\left(\bigwedge_{i \in J} a_{i}\right)^{\prime}=\bigvee_{i \in J} a_{i}^{\prime}$.

Remarks 3.3. (a) Let $a$ be complemented with complement $\neg a$. Then, for every $b$, we have:

(6) $a \backslash b=a \wedge b^{\prime}$.

(7) $b \backslash a=b \wedge \neg a$. 
Indeed: (6) $b \vee\left(a \wedge b^{\prime}\right)=b \vee a \geqslant a$; moreover, if $x \vee b \geqslant a$ then

$$
x \vee b \vee \neg a=1 \Rightarrow x \vee \neg a \geqslant b^{\prime} \Rightarrow(x \vee \neg a) \wedge a \geqslant a \wedge b^{\prime} \Rightarrow x \geqslant x \wedge a \geqslant a \wedge b^{\prime} .
$$

(7) $(b \wedge \neg a) \vee a=b \vee a \geqslant b$; on the other hand,

$$
x \vee a \geqslant b \Rightarrow(x \vee a) \wedge \neg a \geqslant b \wedge \neg a \Rightarrow x \geqslant x \wedge \neg a \geqslant b \wedge \neg a .
$$

This shows how the co-Heyting operation mimics the set difference $B \backslash A$ in the Boolean algebra $\mathcal{P}(X)$.

(b) Under some condition of existence of complements, we have another formula of settheoretical differences:

(8) $a \backslash(b \backslash c)=(a \wedge c) \vee(a \backslash b)$ whenever $c$ is complemented.

Indeed: By the previous remark, $a \backslash(b \backslash c)=a \backslash(b \wedge \neg c)$ while $(a \wedge c) \vee(a \backslash b)=$ $(a \backslash \neg c) \vee(a \backslash b)$. Now apply identity (3.5).

(c) It might be added that Boolean algebras are precisely the co-Heyting algebras in which $a^{\prime \prime}=a$ for every $a$ (as already menioned, in any Boolean algebra $B$ the operator $b \backslash a=b \wedge \neg a$ is a co-Heyting operation; conversely, if $a^{\prime \prime}=a$ for all $a \in B$, then $a \wedge a^{\prime}=\left(a \wedge a^{\prime}\right)^{\prime \prime}=\left(a^{\prime} \vee a^{\prime \prime}\right)^{\prime}=0$ by Proposition 3.2).

(d) For any coframe homomorphism $f: L \rightarrow M$ (i.e. a map between coframes that preserves arbitrary meets and finite joins), $f\left(a^{\prime}\right) \geqslant f(a)^{\prime}$ for every $a \in L$ (because $f\left(a^{\prime}\right) \vee$ $f(a)=f(1)=1)$.

Proposition 3.4. Let $L$ be a complete co-Heyting algebra and $a, b, x \in L$. If $a \wedge x=b \wedge x$ then $a \vee x^{\prime}=b \vee x^{\prime}$.

Proof. It is a consequence of the following obvious fact:

$$
(a \wedge x) \vee x^{\prime}=(b \wedge x) \vee x^{\prime} \quad \text { iff } \quad a \vee x^{\prime}=b \vee x^{\prime} .
$$

We recall that a lattice $L$ is subfit resp. weakly subfit if

$$
a 末 b \text { in } L \quad \Rightarrow \quad \exists c \in L(a \vee c=1 \neq b \vee c)
$$

resp.

$$
a \$ 0 \text { in } L \quad \Rightarrow \quad \exists c \in L(a \vee c=1 \neq c) .
$$

Using (3.7), it is clear that weak subfitness means precisely that

$$
a^{\prime}=1 \quad \Leftrightarrow \quad a=0
$$

(this is the dual property of (4) in Proposition 3.2).

Under co-subfitness conditions, there are surprising formulas for pseudodifferences as certain joins (see [30, Prop. 6.1] for the proof of the dual result): 
Proposition 3.5. In a complete co-Heyting algebra $L$, the formula

$$
b \backslash a=\bigvee\{x \mid a \wedge x=0, x \leqslant b\}
$$

for the co-Heyting operation holds if and only if the dual lattice $L^{o p}$ is subfit.

The formula

$$
a^{\prime}=\bigvee\{x \mid a \wedge x=0\}
$$

for pseudodifference holds if and only if $L^{o p}$ is weakly subfit.

Proposition 3.6. Let $L$ be a complete co-Heyting algebra such that $L^{o p}$ is subfit. Then, for any $a, b \in L$ and any complemented $c$,

$$
c \wedge(b \backslash a)=(c \wedge b) \backslash a .
$$

Proof. Using (3.8) and the well-known fact that in any distributive lattice each complemented element $c$ satisfies the distributivity law $c \wedge \bigvee S=\bigvee\{c \wedge s: s \in S\}$ for any subset $S$, we have $c \wedge(b \backslash a)=\bigvee\{c \wedge x: x \wedge a=0, x \leqslant b\} \leqslant \bigvee\{y: y \wedge a=0, y \leqslant c \wedge b\}=$ $(c \wedge b) \backslash a$.

The next result was first proved by Plewe in [32, Lemma 1.1] for coframes of sublocales but it is indeed a general result on coframes.

Proposition 3.7. Let $L$ be a complete co-Heyting algebra such that $L^{o p}$ is weakly subfit. Then, for any $x \in L$ and any complemented $c$ and $d$,

$$
c \vee x=d \vee x \quad \text { iff } \quad c \backslash x=d \backslash x .
$$

Proof. $\Leftarrow: c \vee x=(c \vee x) \wedge\left(x^{\prime} \vee x\right)=\left(c \wedge x^{\prime}\right) \vee x=\left(d \wedge x^{\prime}\right) \vee x=d \vee x$.

$\Rightarrow$ : Now, using (3.9) we get

$$
c \backslash x=c \wedge x^{\prime}=c \wedge \bigvee\{t \mid t \wedge x=0\}=\bigvee\{c \wedge t \mid t \wedge x=0\}
$$

But, for each such $t, c \wedge t=(c \wedge t) \vee(t \wedge x)=(c \vee x) \wedge t=(d \vee x) \wedge t=d \wedge t$. Hence $c \backslash x=\bigvee\{d \wedge t \mid t \wedge x=0\}=d \wedge x^{\prime}=d \backslash x$.

\section{Coframes of sublocales}

A sublocale of a locale $L$ is a subset $S \subseteq L$ closed under arbitrary meets such that

$$
\forall x \in L \quad \forall s \in S \quad(x \rightarrow s \in S) .
$$

The set $\mathcal{S}(L)$ of all sublocales of $L$ forms a coframe under inclusion (see [29, Th. III.3.2.1] for a proof), in which arbitrary infima coincide with intersections, $\{1\}$ is the bottom element and $L$ is the top element (that we simply denote by 0 and 1 , respectively). Regarding suprema, there is the formula

$$
\bigvee_{i \in I} S_{i}=\left\{\bigwedge A: A \subseteq \bigcup_{i \in I} S_{i}\right\}
$$

for every $\left\{S_{i} \in \mathcal{S}(L): i \in I\right\}$. 
For any $a \in L$, the sets

$$
\mathfrak{c}_{L}(a)=\uparrow a=\{x \in L \mid x \geqslant a\} \text { and } \mathfrak{o}_{L}(a)=\{a \rightarrow b \mid b \in L\}
$$

are the closed and open sublocales of $L$, respectively (that we shall denote simply by $\mathfrak{c}(a)$ and $\mathfrak{o}(a)$ when there is no danger of confusion). For each $a \in L, \mathfrak{c}(a)$ and $\mathfrak{o}(a)$ are complements of each other in $\mathcal{S}(L), \bigcap_{i} \mathfrak{c}\left(a_{i}\right)=\mathfrak{c}\left(\bigvee_{i} a_{i}\right), \mathfrak{c}(a) \vee \mathfrak{c}(b)=\mathfrak{c}(a \wedge b)$, $\bigvee_{i} \mathfrak{o}\left(a_{i}\right)=\mathfrak{o}\left(\bigvee_{i} a_{i}\right)$ and $\mathfrak{o}(a) \cap \mathfrak{o}(b)=\mathfrak{o}(a \wedge b)$

Recall the following basic facts about sublocales $S$ of $L$ (cf. [29]):

(F1) $\mathcal{S}(S)=\{T \cap S \mid T \in \mathcal{S}(L)\}$ and lattice operations in $\mathcal{S}(S)$ are given by those in $\mathcal{S}(L)$ (the only difference is that the two lattices may have different top elements).

(F2) For any open (resp. closed) sublocale $U$ of $L, U \cap S$ is an open (resp. closed) sublocale of $S$. More specifically, for $U=\mathfrak{o}_{L}(a)$ (resp. $U=\mathfrak{c}_{L}(a)$ ), $U \cap S=$ $\mathfrak{o}_{S}\left(\nu_{S}(a)\right)$ (resp. $U \cap S=\mathfrak{c}_{S}\left(\nu_{S}(a)\right)$, where $\nu_{S}(a)=\bigwedge\{s \in S \mid s \geqslant a\}$.

(F3) If $T$ is an open (resp. closed) sublocale of $S$, then $T=U \cap S$ for some $U=\mathfrak{o}(a)$ (resp. $U=\mathfrak{c}(a))$ with $a \in S$.

(F4) Each $S \in \mathcal{S}(L)$ is an intersection of complemented sublocales, specifically

$$
S=\bigcap\left\{\mathfrak{c}(a) \vee \mathfrak{o}(b) \mid \nu_{S}(a)=\nu_{S}(b), a \geqslant b\right\} .
$$

Note that (F4) means that the dual of $\mathcal{S}(L)$ is a zero-dimensional frame and therefore a subfit frame. Hence, all the formulas for pseudodifferences from the preceding section, valid in any coframe whose dual frame is subfit, specialize to the following formulas in $\mathcal{S}(L)$ :

Proposition 4.1. For any $A, B \in \mathcal{S}(L)$ and any complemented $C, D \in \mathcal{S}(L)$ we have:

(1) $B \backslash A=\bigvee\{S \in \mathcal{S}(L) \mid A \cap S=0, S \subseteq B\}$.

(2) $B \backslash A=\bigcap\{S \in \mathcal{S}(L) \mid S$ complemented, $B \subseteq S \vee A\}$.

(3) $B \backslash A=\bigvee\left\{\mathfrak{c}(a) \cap \mathfrak{o}(b) \cap B \mid \nu_{A}(a)=\nu_{A}(b), a \leqslant b\right\}$.

(4) $L \backslash A=\bigvee\{S \in \mathcal{S}(L) \mid A \cap S=0\}$.

(5) $L \backslash A=\bigcap\{S \in \mathcal{S}(L) \mid S$ complemented, $S \vee A=1\}$.

(6) $L \backslash A=\bigvee\left\{\mathfrak{c}(a) \cap \mathfrak{o}(b) \mid \nu_{A}(a)=\nu_{A}(b), a \leqslant b\right\}$

(7) $C \cap(A \backslash B)=(C \cap A) \backslash B$.

(8) $C \vee A=D \vee A$ iff $C \cap(L \backslash A)=D \cap(L \backslash A)$.

(9) $A \subseteq B$ iff for any complemented $C \in \mathcal{S}(L), B \subseteq C \Rightarrow A \subseteq C$.

(10) $L \backslash A \subseteq L \backslash B$ iff for any complemented $C \in \mathcal{S}(L), C \vee B=1 \Rightarrow C \vee A=1$. 
Proof. (1) follows from (3.8), and (2) can be derived from (3.2) and the fact that every sublocale is an intersection of complemented ones.

(3): Using (F4) and (3.5) one gets

$$
B \backslash A=\bigvee\left\{B \backslash(\mathfrak{c}(a) \vee \mathfrak{o}(b)) \mid \nu_{A}(a)=\nu_{A}(b), a \geqslant b\right\}
$$

and then, by property (7) in Remark 3.3 ,

$$
B \backslash A=\bigvee\left\{\mathfrak{c}(b) \cap \mathfrak{o}(a) \cap B \mid \nu_{A}(a)=\nu_{A}(b), a \geqslant b\right\}
$$

(4) is a particular case of (1), (5) is a particular case of (2), (6) is a particular case of (3), while (7) resp. (8) is just Proposition 3.6 resp. 3.7 applied to $\mathcal{S}(L)$.

(9): The implication " $\Rightarrow$ " is obvious. Conversely, let $B=\bigcap_{i \in I} C_{i}$ for some complemented $C_{i}$. Then $A \subseteq C_{i}$ for every $i$, that is, $A \subseteq B$.

Finally, (10) follows immediately from (9).

Remarks 4.2. (a) Regarding property (9) above, note that, on the other hand, the condition $(C \subseteq A \Rightarrow C \subseteq B)$ for every complemented $C$ in $\mathcal{S}(L)$ does not necessarily imply that $A \subseteq B$.

(b) It is clear from formulas (1) and (3) above that for sublocales $A \subseteq B$ of $L, B \backslash A$ calculated in $\mathcal{S}(B)$ (that is, the pseudodifference $A^{\prime}$ in $\mathcal{S}(B)$ ) coincides with $B \backslash A$ calculated in $\mathcal{S}(L)$.

(c) Regarding property (8) of Remark 3.3(b), there is not much hope to improve it in $\mathcal{S}(L)$. Indeed, if the formula holds for any $A, B, C$, the case $A=B=L$ would mean that $C^{\prime \prime}=C$ and thus, by Remark 3.3(c), that $\mathcal{S}(L)$ would be Boolean.

(d) Let $S \in \mathcal{S}(L)$. By (P2), $L \backslash S=0$ iff $S=L$. On the other hand, there might exist nonzero $S$ such that $L \backslash S=L$. It is easy to check, using 4.1(5), that these are precisely the sublocales $S$ that contain no nonzero complemented sublocales, introduced by $\mathrm{T}$. Plewe [33] as the rare sublocales.

(e) [31] has a few more special formulas for the supplements in particular classes of frames like the $T_{1}$-spatial or subfit ones, where they are used to show that the system of all joins of closed sublocales of $L$ is the Booleanization of $\mathcal{S}(L)$. Recently, in [16], T. Dube uses these formulas to compute remainders $\beta L \backslash L, \beta L \backslash v L$ and $\beta L \backslash \lambda L$ for any completely regular $L$ (see Section 7 below for details about $v L$ and $\lambda L$ ).

For each sublocale $A$ of $L$, the closure and interior of $A$ are defined, respectively, as $\operatorname{cl}_{L} A=\bigcap\{\mathfrak{c}(a) \mid A \subseteq \mathfrak{c}(a)\}$ and $\operatorname{int}_{L} A=\bigvee\{\mathfrak{o}(a) \mid \mathfrak{o}(a) \subseteq A\}$. We shall write $\mathrm{cl} A$ instead of $\operatorname{cl}_{L} A$ (and, similarly, for the interior) when there is no danger of confusion about the ambient frame. It is clear that $\operatorname{cl} A=\mathfrak{c}(\bigwedge A)$; on the other hand, since $\mathfrak{o}(a) \subseteq A$ if and only if $A^{\prime} \subseteq \mathfrak{c}(a)$, we have int $A=\mathfrak{o}\left(\bigwedge A^{\prime}\right)$. In particular, cl $\mathfrak{o}(a)=\mathfrak{c}\left(a^{*}\right)$ and $\operatorname{int} \mathfrak{c}(a)=\mathfrak{o}\left(a^{*}\right)$.

It then follows that $\operatorname{int} A^{\prime \prime}=\operatorname{int} A$ and their complement is

$$
\operatorname{cl}(L \backslash A)=\operatorname{cl} A^{\prime}=\mathfrak{c}\left(\bigwedge A^{\prime}\right)=L \backslash \operatorname{int} A .
$$

Note, however, that the corresponding formula for the interior does not hold generally:

$$
\operatorname{int}(L \backslash A)=\mathfrak{o}(\bigwedge A) \subseteq \mathfrak{o}\left(\bigwedge A^{\prime \prime}\right)=L \backslash \operatorname{cl} A .
$$


In fact, from (4.2) it only follows that

$$
\operatorname{int}(L \backslash A)=L \backslash \operatorname{cl}(L \backslash(L \backslash A))=L \backslash \operatorname{cl} A^{\prime \prime} .
$$

In summary, the interior and closure operators generate from any sublocale $A$ the following three towers of inclusions (the arrow $S<\cdots>T$ indicates that $S$ and $T$ are complemented to each other):

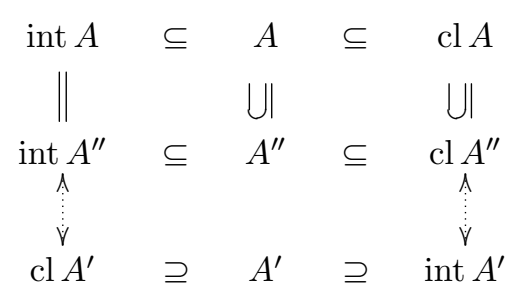

\section{Images, preimages and pseudodifferences}

Let $f: L \rightarrow M$ be a localic map. The image $f[S]$ of any sublocale $S \subseteq L$ is a sublocale of $M$ and we have the localic image function

$$
f[-]: \mathcal{S}(L) \rightarrow \mathcal{S}(M) .
$$

On the other hand, the set-theoretic preimage $f^{-1}[S]$ of a sublocale $S$ is not necessarily a sublocale. It is a subset closed under meets, though, and hence, by the formula (4.1), there is the largest sublocale

$$
f_{-1}[S]=\bigvee\left\{T \in \mathcal{S}(L) \mid T \subseteq f^{-1}[S]\right\}
$$

contained in $f^{-1}[S]$. This defines the localic preimage function

$$
f_{-1}[-]: \mathcal{S}(M) \rightarrow \mathcal{S}(L),
$$

right adjoint of $f[-]$ (that is, $f[S] \subseteq T$ if and only if $S \subseteq f_{-1}[T]$ ). Note that $f_{-1}[S]$ is the pullback in Loc of $S$ along $f$. For closed sublocales we have $f_{-1}[\mathfrak{c}(a)]=f^{-1}[\mathfrak{c}(a)]=$ $\mathfrak{c}\left(f^{*}(a)\right)$. For open sublocales the localic and set-theoretic preimages do not necessarily coincide, but we do have $f_{-1}[\mathfrak{o}(a)]=\mathfrak{o}\left(f^{*}(a)\right)$.

The preimage function is a coframe homomorphism (that preserves complements) while $f[-]$ is a colocalic map $([29])$. Hence the latter satisfies the dual properties of (L1)-(L3) in Section 2:

$\left(\mathrm{L}^{\prime}\right) f\left[\bigvee_{i \in I} S_{i}\right]=\bigvee_{i \in I} f\left[S_{i}\right]$ (in particular, $f[0]=0$ ).

$\left(\mathrm{L}^{\prime}\right) f\left[S \backslash f_{-1}[T]\right]=f[S] \backslash T$.

$\left(\mathrm{L} 3^{\prime}\right) f[S]=0 \Rightarrow S=0$.

On the other hand, the preimage being a coframe homomorphism satisfies

$$
L \backslash f_{-1}[T] \subseteq f_{-1}[M \backslash T] .
$$


Remarks 5.1. (a) By $\left(\mathrm{L1}^{\prime}\right), f[L \backslash S]=\bigvee\{f[R] \mid R \cap S=0\}$. So, the inclusion $f[L \backslash S] \subseteq M \backslash T$ means that

$$
\bigvee\{f[R] \mid R \cap S=0\} \subseteq \bigcap\{V \mid V \vee T=1\},
$$

that is, for every $R \in \mathcal{S}(L)$ and $V \in \mathcal{S}(M), R \cap S=0$ and $V \vee T=1$ imply $f[R] \subseteq V$.

(b) The case $S=L$ in (L2') says that

$$
f\left[L \backslash f_{-1}[T]\right]=f[L] \backslash T \subseteq M \backslash T .
$$

Thus

$$
f\left[L \backslash f_{-1}[T]\right]=M \backslash T
$$

whenever $f$ is onto.

(c) By (5.1), $f\left[L \backslash f_{-1}[T]\right] \subseteq f f_{-1}[M \backslash T] \subseteq M \backslash T \subseteq M \backslash f\left[f_{-1}[T]\right]$. Hence, for any sublocale $S$ of $L$ which is the preimage of some sublocale $T$ of $M$,

$$
f[L \backslash S] \subseteq M \backslash f[S] .
$$

(d) The reverse inclusion of (5.4) holds for any sublocale $S$ provided $f$ is onto. Indeed, we have $S \subseteq f_{-1}[f[S]]$, thus $L \backslash f_{-1}[f[S]] \subseteq L \backslash S$ and consequently (using (5.3) for $T=f[S])$

$$
M \backslash f[S]=f\left[L \backslash f_{-1}[f[S]]\right] \subseteq f[L \backslash S] .
$$

\section{Remainders}

Recall that a sublocale $S$ of a locale $L$ is dense precisely when $0 \in S$, since $\bar{S}=\uparrow(\bigwedge S)$ is all of $L$ if and only if $0=\bigwedge S \in S$. It follows from formula (4.2) that $L \backslash A$ is dense iff int $A=0$.

Remark 6.1. For sublocales $S \subseteq T \subseteq L$, if $T$ is dense in $L$ and $S$ is closed in $L$, then $T \backslash S$ is dense in $L \backslash S$. In fact, if $S=\mathfrak{c}_{L}(a)$ for some $a \in T$, then $T \backslash S=\mathfrak{o}_{T}(a)$ while $L \backslash S=\mathfrak{o}_{L}(a)$; in particular, $0_{T \backslash S}=a \rightarrow 0_{T}=a \rightarrow 0_{L}=0_{L \backslash S}$ since $0_{T}=0_{L}$.

Recall further that a compactification of a locale $L$ is a compact regular locale $M$ together with a dense localic embedding $\kappa: L \longmapsto M$. Being dense means that $\kappa[L]$ is dense in $M$, that is, $\kappa(0)=0$. For general background on compactifications of frames and locales the reader is referred to Banaschewski [5].

It seems now appropriate to introduce the following definition:

Definition 6.2. For any compactification $\kappa: L \longmapsto M$, the remainder of $L$ in the compactification is the sublocale $M \backslash \kappa[L]$ of $M$. Sometimes, when no confusion is possible, we shall simply denote the sublocale $\kappa[L]$ of $M$ by $L$ and its remainder in $M$ by $M \backslash L$.

Let us mention that this notion appeared already in the literature in a paper by $\mathrm{D}$. Baboolal [1], formulated for the Freudenthal compactification in terms of its frame of congruences but it is readily seen to be equivalent to the definition above.

Let us compute remainders in some illustrative examples: 


\section{(A) Alexandroff compactification $([2,3,5])$.}

Let $\kappa: L \longmapsto M$ be a compactification of a locally compact (i.e., continuous) frame $L$ and let $m_{L}=\bigvee\{\kappa(x) \mid x \ll 1\}$. Since $\kappa$ is an embedding, that is, $\kappa^{*} \kappa=1$, we have $\kappa^{*}\left(m_{L}\right)=\bigvee_{x \ll 1} x=1$ by continuity. It follows that $\kappa[L] \subseteq \mathfrak{o}\left(m_{L}\right)$ : for every $x \in L$,

$$
\kappa(x)=\kappa(1 \rightarrow x)=\kappa\left(\kappa^{*}\left(m_{L}\right) \rightarrow x\right)=m_{L} \rightarrow \kappa(x) .
$$

Hence $M \backslash L \supseteq \mathfrak{c}\left(m_{L}\right)$ and we may regard $\kappa$ as a localic embedding

$$
\kappa: L \longmapsto \mathfrak{o}\left(m_{L}\right) .
$$

If one assumes moreover that $L$ is regular, one can say more. Indeed, as proved in [2, Th. $2.2]$, in that case $\kappa^{*}: \mathfrak{o}\left(m_{L}\right) \rightarrow L$ is a codense homomorphism, that is, $\kappa^{*}(x)=1 \Rightarrow x=1$.

Remark 6.3. In localic terms, this means that $\kappa[L]$ is a codense sublocale of $\mathfrak{o}\left(m_{L}\right)$ ([15], called replete in [30]), that is, $\mathfrak{c}(a) \cap \kappa[L] \neq 0$ for every $a \neq 1$ in $\mathfrak{o}\left(m_{L}\right)$.

Lemma 6.4. Let $f: L \rightarrow M$ be a localic map with $M$ regular and $f[L]$ codense in $M$. Then $f$ is onto.

Proof. Let $y$ be an arbitrary element of $M$ and $x=f f^{*}(y)$. By regularity, $x=\bigvee\{z \mid$ $z<x\}$. For each such $z$ we have $1=f^{*}\left(z^{*}\right) \vee f^{*}(x)=f^{*}\left(z^{*}\right) \vee f^{*}(y)=f^{*}\left(z^{*} \vee y\right)$. Since $f[L]$ is codense in $M$, this implies $z<y$. Hence $y=x=f f^{*}(y) \in f[L]$.

Applying this property to our $\kappa: L \longmapsto \mathfrak{o}\left(m_{L}\right)$ we get immediately the following:

Proposition 6.5. Let $\kappa: L \longmapsto M$ be a compactification of a regular continuous frame $L$. Then $\kappa[L]=\mathfrak{o}\left(m_{L}\right)$ and therefore $M \backslash L=\mathfrak{c}\left(m_{L}\right)$.

Of course, if $L$ is non-compact then $m_{L}<1$ and $M \backslash L \neq 0$.

\section{(B) Stone-Čech compactification ([8]).}

A crucial example in this context is the pointfree Stone-Cech compactification, introduced by Banaschewski and Mulvey in [8]. It establishes a reflection of the category of completely regular locales into the (full) subcategory of compact, completely regular locales. We recall it briefly here. Let $\Im(L)$ be the poset of all ideals of $L$ (ordered by inclusion). Its top element is $L$ while $\{0\}$ is the bottom element. Since any intersections of ideals is an ideal, $\mathfrak{I}(L)$ is a complete lattice with (arbitrary) meets given by intersections. The joins are given by the formula

$$
\bigvee_{\alpha} I_{\alpha}=\left\{\bigvee F \mid F \subseteq \bigcup_{\alpha} I_{\alpha}, F \text { finite }\right\}
$$

It follows from this formula that $\mathfrak{I}(L)$ is a compact frame.

Now assume that $L$ is completely regular: for each $a \in L, a=\bigvee\{x \in L \mid x<<a\}$. An $I \in \mathfrak{I}(L)$ is called regular (with respect to the strong relation $\prec<$ ) if it satisfies the condition

$$
\forall a \in I \quad \exists b \in I \quad(a<<b) .
$$

Examples of regular ideals are the $\downarrow a=\{x \in L \mid x<<a\}$ for any $a \in L$. The collection $\mathfrak{R}(L)$ of all regular ideals is a subframe of $\mathfrak{I}(L)$ hence a compact frame. It is also easily 
shown to be regular and hence, being compact, completely regular. The Stone-Čech compactification of $L$ is the dense localic embedding

$$
\beta_{L}: L \longmapsto \beta L=\mathfrak{R}(L)
$$

given by $\beta_{L}(a)=\downarrow a$. Its left adjoint is the frame homomorphism $\beta_{L}^{*}: I \mapsto \bigvee I$. The following properties are well known:

(a) $\beta_{L}^{*} \beta_{L}=\mathrm{id}_{L}$.

(b) $\forall I \in \beta L, I=\bigvee\left\{\beta_{L}(a) \mid a \in I\right\}$ (by formula (6.1)).

(c) $\beta_{L}\left(a^{*}\right)=\beta_{L}(a)^{*}, I^{*}=\beta_{L}\left((\bigvee I)^{*}\right)$.

(d) $\beta_{L}$ preserves $<<$ and $\beta_{L}(a)<I$ iff $a \in I$.

Examples 6.6. (1) In any Boolean frame, every ideal is regular (since $x<x$ for every complemented $x$ ). Hence $\beta L=\mathfrak{R}(L)=\mathfrak{I}(L)$ for any Boolean $L$. In particular, for $L=\mathbf{2}$, $\beta L=\{\downarrow 0, \downarrow 1\} \simeq \mathbf{2}$.

(2) For finite locales $L$, every ideal is principal. In this case, $L$ being completely regular makes it Boolean, so that $\beta L$ is isomorphic to $L$.

(3) For any compact completely regular locale $L, \beta_{L}: L \rightarrow \beta L$ is an isomorphism and thus $\beta L=\beta_{L}(L) \cong L([8])$.

The corresponding functor $\beta$ : CRegLoc $\rightarrow$ KCRegLoc (that shows that the category KCRegLoc of compact completely regular locales is a reflective subcategory of CRegLoc) is defined as follows:

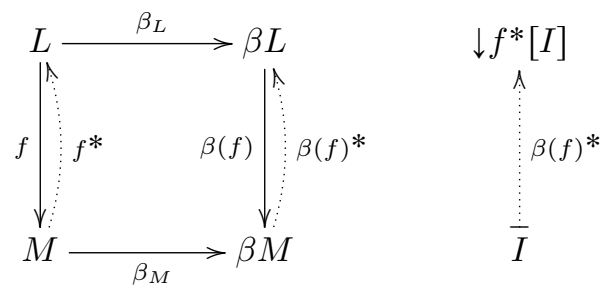

We call $\beta(f): \beta L \rightarrow \beta M$ the Stone extension of $f$. It is defined directly by

$$
\beta(f)(J)=\bigvee\left\{I \in \beta M \mid \beta(f)^{*}(I) \subseteq J\right\}=\bigvee\left\{I \in \beta M \mid f^{*}[I] \subseteq J\right\} .
$$

By 4.1(4), the remainder of $L$ in $\beta L$ is given by

$$
\beta L \backslash L=\bigvee\left\{S \in \mathcal{S}(\beta L) \mid S \cap \beta_{L}[L]=0\right\}=\bigvee\{S \in \mathcal{S}(\beta L) \mid \downarrow a \in S \Rightarrow a=1\}
$$

Note that

$$
\beta L \backslash L=0 \quad \text { iff } \quad L=\beta L
$$

but, on the other hand, $\beta L \backslash L=\beta L$ does not imply in general that $L=0$ (only the converse implication holds).

\section{(C) Freudenthal compactification ([1]).}

A regular frame $L$ is called rim-compact ([1]) if each $a \in L$ is a join of elements $u$ such that $\mathfrak{c}\left(u \vee u^{*}\right)$ is compact. A basis $B$ of a frame $L$ is a $\pi$-compact basis if, for every $a, b \in B, \mathfrak{c}\left(a \vee a^{*}\right)$ is compact, $a^{*} \in B, a \wedge b \in B$ and $a \vee b \in B$. 
Remark 6.7. It is easy to check that in any rim-compact frame $L$, the subset $B_{0}=\{b \in$ $L \mid \mathfrak{c}\left(b \vee b^{*}\right)$ is compact $\}$ is a $\pi$-compact basis.

Given a $\pi$-compact basis $B$ for a rim-compact frame $L$, define a relation $\triangleleft_{B}$ on $L$ by $a \triangleleft_{B} b$ if and only if there exists $u \in B$ such that $a \prec u<b$. This is a strong relation on $L([5])$ and hence establishes a compactification

$$
\gamma_{L}^{B}: L \longmapsto \gamma_{B} L .
$$

Here $\gamma_{B} L$ is the frame of ideals of $L$ regular with respect to $\triangleleft_{B}$ (i.e., the ideals that satisfy a similar condition to (6.2) for $\triangleleft_{B}$ ), and $\gamma_{L}^{B}(a)=\left\{x \in L \mid x \triangleleft_{B} a\right\}$. The left adjoint $\left(\gamma_{L}^{B}\right)^{*}$ is again given by joins: $\left(\gamma_{L}^{B}\right)^{*}(I)=\bigvee I$. Note that $\gamma_{L}^{B}[B]=\left\{\gamma_{B}(b) \mid b \in B\right\}$ is a basis for $\gamma_{B} L$.

This is the $\pi$-compactification of $L$ induced by basis $B$. The Freudenthal compactification of $L$ ([1]) is just the compactification $\gamma_{L}^{B_{0}}$ induced by the basis $B_{0}$ of the remark above, that we denote as $\gamma_{L}: L \longmapsto \gamma L$.

Remark 6.8. Like the Stone-Čech compactification, this is an example of a perfect compactification ([1]), i.e. $\gamma_{L}\left(u \vee u^{*}\right)=\gamma_{L}(u) \vee \gamma_{L}\left(u^{*}\right)$ for every $u \in L$.

Proposition 6.9. Let $\gamma_{L}: L \longmapsto \gamma L$ be the Freudenthal compactification of a rim-compact frame $L$. Then $\gamma L \backslash L$ is a zero-dimensional sublocale of $\gamma L$.

Proof. We prove it in two steps:

(1) $\left\{j_{L}^{*} \gamma_{L}(b) \mid b \in B_{0}\right\}$ is a basis for $\gamma L \backslash L$ (where $j_{L}$ denotes the sublocale embedding $\gamma L \backslash L \longmapsto \gamma L)$;

(2) Each $j_{L}^{*} \gamma_{L}(b)\left(b \in B_{0}\right)$ is complemented in $\gamma L \backslash L$.

Let us proceed with it:

(1) This is an immediate consequence of the fact that $\gamma_{L}\left[B_{0}\right]$ is a basis for $\gamma L$. In fact, each $a \in \gamma L \backslash L$ is in $\gamma L$ thus there are some $b_{i} \in B_{0}(i \in I)$ such that $a=\bigvee_{i \in I} \gamma_{L}\left(b_{i}\right)$; then $a=j_{L}^{*}(a)=\bigvee_{i \in I} j_{L}^{*} \gamma_{L}\left(b_{i}\right)$.

(2) First, for each $b \in B_{0}$ we have

$$
j_{L}^{*} \gamma_{L}(b) \wedge j_{L}^{*} \gamma_{L}\left(b^{*}\right)=j_{L}^{*}\left(\gamma_{L}(b) \wedge \gamma_{L}\left(b^{*}\right)\right)=j_{L}^{*} \gamma_{L}(0)=0
$$

(since $\gamma_{L}$ is dense). Moreover,

$$
j_{L}^{*} \gamma_{L}(b) \vee j_{L}^{*} \gamma_{L}\left(b^{*}\right)=j_{L}^{*}\left(\gamma_{L}(b) \vee \gamma_{L}\left(b^{*}\right)\right)=j_{L}^{*} \gamma_{L}\left(b \vee b^{*}\right)
$$

(because $\gamma_{L}$ being perfect, preserves disjoint binary joins [1, Theorem 3.5(3)]). It suffices now to check that $j_{L}^{*} \gamma_{L}\left(b \vee b^{*}\right)$ is the top element of the remainder $\gamma L \backslash L$, that is, $1_{\gamma L}$. By the definition of $j_{L}^{*}$, the image $j_{L}^{*} \gamma_{L}\left(b \vee b^{*}\right)$ is the element $\nu_{\gamma L \backslash L}\left(\gamma_{L}\left(b \vee b^{*}\right)\right)$ of $\gamma L \backslash L$. Let us consider the restriction of $\gamma_{L}: L \rightarrow \gamma L$ to the closed part $\mathfrak{c}\left(b \vee b^{*}\right)$. This is clearly an embedding

$$
\left.\gamma_{L}\right|_{\mathfrak{c}\left(b \vee b^{*}\right)}: \mathfrak{c}\left(b \vee b^{*}\right) \longmapsto \mathfrak{c}\left(\gamma_{L}\left(b \vee b^{*}\right)\right),
$$

a dense one obviously. In addition, $\mathfrak{c}\left(b \vee b^{*}\right)$ is compact (by the definition of $\left.B_{0}\right)$ and regular (as a sublocale of a regular locale); $\mathfrak{c}\left(\gamma_{L}\left(b \vee b^{*}\right)\right)$ is compact (because it is a 
closed sublocale of a compact locale) and regular too. In conclusion, $\left.\gamma_{L}\right|_{\mathfrak{c}\left(b \vee b^{*}\right)}$ is a dense embedding between compact regular locales, hence an isomorphism. In particular, $\gamma[L] \supseteq \mathfrak{c}\left(\gamma_{L}\left(b \vee b^{*}\right)\right)$. Now, the required fact that $\nu_{\gamma L \backslash L}\left(\gamma_{L}\left(b \vee b^{*}\right)\right)=1$ is clear: if $s \in \gamma L \backslash L$ with $s \geqslant \gamma_{L}\left(b \vee b^{*}\right)$, then $s \in \gamma L \backslash \mathfrak{c}\left(\gamma_{L}\left(b \vee b^{*}\right)\right)=\mathfrak{o}\left(\gamma_{L}\left(b \vee b^{*}\right)\right)$; hence $s \in \mathfrak{c}\left(\gamma_{L}\left(b \vee b^{*}\right)\right) \cap \mathfrak{o}\left(\gamma_{L}\left(b \vee b^{*}\right)\right)=0$, that is, $s=1$.

\section{Remainder preservation}

In this section, except when otherwise noted, all our frames (locales) are completely regular.

Let $f: L \rightarrow M$ be a localic map between completely regular locales, $S$ a sublocale of $L$ and $T$ a sublocale of $M$. We say that $f$ takes the remainder of $S$ to the remainder of $T$ (briefly, takes $S$-remainder to $T$-remainder) if

$$
f[L \backslash S] \subseteq M \backslash T
$$

that is,

$$
L \backslash S \subseteq f_{-1}[M \backslash T] .
$$

In particular, when the Stone extension $\beta(f): \beta L \rightarrow \beta M$ of $f$ takes the remainder of $\beta[L]$ to the remainder of $\beta[M]$, we simply say that $\beta(f)$ takes remainder to remainder. In that case, the given localic $f$ is said to be $\beta$-remainder preserving. We use a similar terminology for other examples of reflections in the category of completely regular locales.

Evidently, compositions of $\beta$-remainder preserving maps are $\beta$-remainder preserving.

Remark 7.1. Any localic map $f: L \rightarrow M$ with $L$ compact is $\beta$-remainder preserving. In fact, if $L$ is compact, that is, $\beta L=L$, then $\beta L \backslash L=0$, by (6.3), and therefore $\beta(f)[\beta L \backslash L]=\beta(f)[0]=0$.

Let $\pi$ be the unique localic map $L \rightarrow \mathbf{2}$ (which is given by $\pi(1)=1$ and $\pi(a)=0$ for every $a \neq 1$ ). In [13], Chen shows that properness of maps characterizes compact locales in the sense that a locale $L$ is compact if and only if $\pi: L \rightarrow \mathbf{2}$ is proper (see Section 8 below for the definition of proper map of locales). Now, we have:

Proposition 7.2. A locale $L$ is compact if and only if $\pi: L \rightarrow \mathbf{2}$ is $\beta$-remainder preserving.

Proof. $\Leftarrow$ : By Example 6.6(1), $\beta \mathbf{2}=\mathbf{2}$. Hence $\beta(\pi)[\beta L \backslash L] \subseteq \beta \mathbf{2} \backslash \mathbf{2}=0$. Then, by $\left(\mathrm{L}^{\prime}\right), \beta L \backslash L=0$ and therefore $L \supseteq \beta L \backslash(\beta L \backslash L)=\beta L$. Hence $L=\beta L$ is compact. $\Rightarrow$ : if $L$ is compact, that is, $\beta L=L$, then $\beta(\pi)=\pi$ and thus $\beta(\pi)[\beta L \backslash L]=\pi[0]=0$.

With $v L$ and $\lambda L$ denoting, respectively, the realcompact reflection and the regular Lindelöf reflection of a completely regular locale $L$, we may also speak about $v$ - and $\lambda$-remainder preserving maps.

The realcompact reflection $v$ is a reflection of the subcategory of realcompact locales in the category of completely regular frames. Recall that a frame $L$ is said to be realcompact [7] if, for any maximal ideal $I$ of $\mathrm{Coz} L$ (the cozero part of $L$ [7]) such that $\bigvee I=1$, there is a countable $S \subseteq I$ such that $\bigvee S=1$. For more details about the construction of the realcompact coreflection $v L$ of an $L$ see [7] (or [17]). 
The regular Lindelöf reflection $\lambda$ (the Lindelöfication), originally constructed by Madden and Vermeer in [28], shows that regular Lindelöf locales form a reflective subcategory of the category of completely regular locales. Note that this is a fact in locales that has no counterpart in the classical setting of topological spaces and continuous maps; in general, $\lambda L$ is not spatial even when $L$ is spatial. The reflection $\lambda L$ of any completely regular locale $L$ is the intersection of all cozero-sublocales of $\beta L$ that contain $L$. For a description of this construction in terms of frames see e.g. [17]. There one may also see that $\beta$ dominates $\lambda$ and $\lambda$ dominates $v$, that is, there are dense embeddings

$$
\ell_{L}: v L \longmapsto \lambda L, \quad \kappa_{L}: \lambda L \longmapsto \beta L .
$$

An inspection of the proof of Proposition 7.2 shows that a similar result holds more generally for any monoreflection $\mathfrak{R}$ on locales such that $\mathfrak{R} \mathbf{2}=\mathbf{2}$. Hence we have immediately:

Corollary 7.3. Let $L$ be a locale: Then:

(1) $L$ is Lindelöf if and only if $\pi: L \rightarrow \mathbf{2}$ is $\lambda$-remainder preserving.

(2) $L$ is realcompact if and only if $\pi: L \rightarrow \mathbf{2}$ is $v$-remainder preserving.

Remark 7.4. The remainder preserving maps treated by Dube and Naidoo in [17] for the extensions $\beta, \lambda$ and $v$ are referred to as, respectively, $\beta$-proper, $\lambda$-proper and $v$ proper. Corollary 7.3 above shows that our notion of remainder preserving maps does not coincide with Dube-Naidoo's one and might be viewed as a more satisfactory one. Indeed, the latter notion of properness cannot distinguish between the preservation of $\lambda$-remainders and $v$-remainders: a localic map is $\lambda$-proper if and only if it is $v$-proper ([17, Proposition 4.4]). This should not come as a surprise regarding the fact that DubeNaidoo's properness definition relies on the points of the locale and the spectrum of $\lambda(\mathfrak{O} X)$ for any space $X$ is precisely $v X([28])$. Our notion, being defined inside the sublocale lattice, is able to distinguish the two cases.

The next result is the localic counterpart to Lemma 4.2 of [17].

Proposition 7.5. Suppose that in the diagram

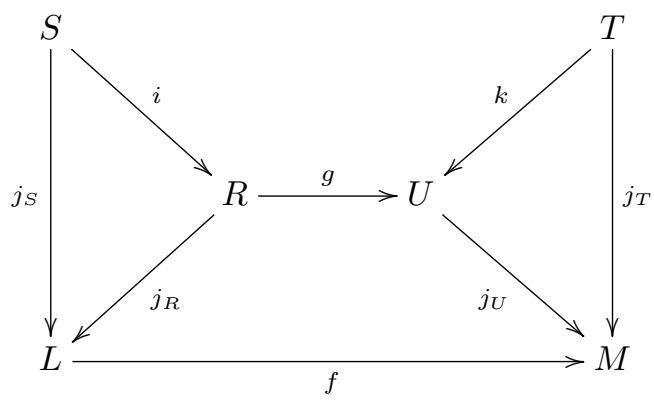

the downward morphisms are embeddings, the triangles commute, the trapezoid commutes and $f$ takes $S$-remainder to $T$-remainder. If one of the following conditions holds then $g$ also takes $S$-remainder to $T$-remainder: 
(a) $U$ is a complemented sublocale of $M$.

(b) $T$ is a complemented sublocale of $M$.

Proof. By hypothesis, $f[L \backslash S] \subseteq M \backslash T$. Then

$$
U \cap(M \backslash T) \supseteq U \cap f[L \backslash S] \supseteq f\left[f_{-1}[U]\right] \cap f[L \backslash S] \supseteq f\left[f_{-1}[U] \cap(L \backslash S)\right] .
$$

In addition, $f[R]=g[R] \subseteq U$, that is, $R \subseteq f_{-1}[U]$. Hence

$$
g[R \backslash S]=f[R \backslash S] \subseteq f[R \cap(L \backslash S)] \subseteq U \cap(M \backslash T) .
$$

Finally:

(a) Under condition (a), we may apply Proposition 4.1(7) to conclude that $g[R \backslash S] \subseteq$ $U \cap(M \backslash T)=U \backslash T$.

(b) On the other hand, under condition (b) we may use property (7) in 3.3 to get $g[R \backslash S] \subseteq U \cap(M \backslash T)=U \cap \neg T=U \backslash T$.

Corollary 7.6. Let $f: L \rightarrow M$ with $M$ complemented in $\beta M$.

(1) If $f$ is $\beta$-remainder preserving then it is $\lambda$-remainder preserving.

(2) If $f$ is $\lambda$-remainder preserving then it is $v$-remainder preserving.

Proof. (1) By (7.1), we have the diagram

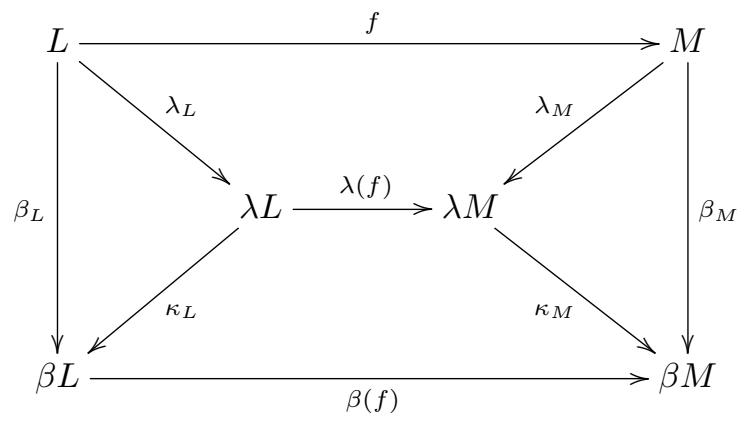

Apply Proposition 7.5.

(2) can be proved in a similar way.

Example 7.7. As an example of a family of locales $M$ satisfying the assumption of Corollary 7.6 we mention the locally compact, completely regular locales. In fact, any locally compact, completely regular locale is an open sublocale (thus complemented) of its Stone-Čech compactification ([29, VII.5.3]). Hence, for any locally compact, completely regular locale $M$ and any localic map $f: L \rightarrow M$,

$f$ is $\beta$-remainder pres. $\Rightarrow f$ is $\lambda$-remainder pres. $\Rightarrow f$ is $v$-remainder pres.

We end this section with several characterizations of remainder preservation by localic maps. 
Proposition 7.8. Let $f: L \rightarrow M$ be a localic map, $S \in \mathcal{S}(L)$ and $T \in \mathcal{S}(M)$. The following are equivalent:

(i) $f$ takes $S$-remainder to $T$-remainder.

(ii) For each $U \in \mathcal{S}(M)$,

$$
U \vee T=1 \Rightarrow f_{-1}[U] \vee S=1 .
$$

(iii) For each complemented $C \in \mathcal{S}(M)$,

$$
C \vee T=1 \Rightarrow f_{-1}[C] \vee S=1 .
$$

(iv) For each complemented $C \in \mathcal{S}(M)$ and each complemented $D \supseteq T$,

$$
C \vee D=1 \Rightarrow f_{-1}[C] \vee E=1
$$

for every complemented $E \supseteq S$.

(v) For each $R \in \mathcal{S}(L)$,

$$
R \cap S=0 \Rightarrow R \subseteq f_{-1}[M \backslash T] .
$$

(vi) For each $R \in \mathcal{S}(L)$ and for each complemented $C \in \mathcal{S}(M)$,

$$
(R \cap S=0, C \vee T=1) \Rightarrow R \subseteq f_{-1}[C] .
$$

Proof. (i) $\Leftrightarrow(i i):$ Since

$$
\begin{aligned}
f_{-1}[M \backslash T] & =f_{-1}[\bigcap\{U \in \mathcal{S}(M) \mid U \vee T=1\}] \\
& =\bigcap\left\{f_{-1}[U] \mid U \in \mathcal{S}(M), U \vee T=1\right\}
\end{aligned}
$$

we have $L \backslash S \subseteq f_{-1}[M \backslash T]$ if and only if $L \backslash S \subseteq f_{-1}[U]$, that is, $f_{-1}[U] \vee S=1$, for each such $U$.

(ii) $\Leftrightarrow$ (iii) and (iii) $\Leftrightarrow$ (iv) follow immediately from characterizations (9) and (10) in Proposition 4.1 .

(i) $\Leftrightarrow(\mathrm{v}): f[L \backslash S] \subseteq M \backslash T \Leftrightarrow f[\bigvee\{R \mid R \cap S=0\}] \subseteq M \backslash T \Leftrightarrow \bigvee\{f[R] \mid R \cap S=$ $0\} \subseteq M \backslash T$ and this is equivalent to $f[R] \subseteq M \backslash T$, that is, $R \subseteq f_{-1}[M \backslash T]$, for each such $R$.

Finally, $(\mathrm{v}) \Leftrightarrow(\mathrm{vi})$ follows immediately from Proposition 4.1(5).

For the sake of completeness let us also mention that our concept of remainder preservation can be treated in the following more general setting. Let $g: A \rightarrow B$ be a localic map with a left adjoint $g^{*}$. For each $x \in A$ and $y \in B, g$ takes $x$-remainder to $y$-remainder if

$$
y^{*} \leqslant g\left(x^{*}\right), \text { that is, } g^{*}\left(y^{*}\right) \leqslant x^{*} .
$$

Note that in this section we have just treated the following two cases:

(1) $A=\mathcal{S}(L)^{o p}, B=\mathcal{S}(M)^{o p}, g=f[-], g^{*}=f_{-1}[-], x=S$ and $y=T$. 
(2) $A=\mathcal{S}(\beta L)^{o p}, B=\mathcal{S}(\beta M)^{o p}, g=\beta(f)[-], g^{*}=\beta(f)_{-1}[-], x=L$ and $y=M$.

For instance, characterizations (v) and (vi) of the preceding proposition are particular cases of assertions (a) and (b) in the following result (which is an easy consequence of Proposition 3.5):

Proposition 7.9. Let $g: A \rightarrow B$ be a localic map. If $A$ is weakly subfit then we have:

(a) $g$ takes $x$-remainder to $y$-remainder if and only if for each $a \in A$,

$$
\left.a \vee x=1 \Rightarrow y^{*} \leqslant g(a) \text { (i.e., } g^{*}(y) \leqslant a\right) \text {. }
$$

(b) Moreover, if $B$ is zero dimensional, then $g$ takes $x$-remainder to y-remainder if and only if for each $a \in A$ and for each complemented $c \in M$,

$$
(a \vee x=1, c \wedge y=0) \Rightarrow g^{*}(c) \leqslant a .
$$

\section{Remainder preserving maps and proper maps}

Recall from [20] (consult [36] for more information) that a localic map $f: L \rightarrow M$ is proper (aka perfect $[24,12]$ ) if it is closed (that is, $f\left(f^{*}(b) \vee a\right)=b \vee f(a)$ for every $a \in L$ and $b \in M$ ) and preserves directed joins. By Theorem 1.1 quoted in the Introduction,

a localic map $f: L \rightarrow M$ between completely regular locales is proper if and

only if $\beta(f)_{-1}[M]=L$.

Proposition 8.1. Any proper localic map is $\beta$-remainder preserving.

Proof. Let $\beta(f)_{-1}[M]=L$. Then $\beta(f)[\beta L \backslash L]=\beta(f)\left[\beta L \backslash \beta(f)_{-1}[M]\right]$, and by property $\left(\mathrm{L} 2^{\prime}\right)$ we get

$$
\beta(f)[\beta L \backslash L]=\beta(f)[\beta L] \backslash M \subseteq \beta M \backslash M .
$$

Let us analyse why, contrarily to what happens in the spatial case, the converse implication does not hold in general. In the pullback condition

$$
\beta(f)_{-1}[M]=L,
$$

the inclusion $L \subseteq \beta(f)_{-1}[M]$ means that $\beta(f)[L] \subseteq M$; on the other hand, the reverse inclusion is just what is needed in the proof of the preceding proposition:

$$
\begin{aligned}
\beta(f)_{-1}[M] \subseteq L \Rightarrow \beta(f)[\beta L \backslash L] & \subseteq \beta(f)\left[\beta L \backslash \beta(f)_{-1}[M]\right] \\
& =\beta(f)[\beta L] \backslash M \subseteq \beta M \backslash M .
\end{aligned}
$$

Hence

$$
\beta(f)_{-1}[M]=L \Rightarrow[\beta(f)[\beta L \backslash L] \subseteq \beta M \backslash M \text { and } \beta(f)[L] \subseteq M] .
$$

What about the converse to Proposition 8.1?

In our situation, we have always $f[L] \subseteq M$, that is, $\beta(f)[L] \subseteq M$, so the equivalence in Proposition 8.1 amounts to the equivalence between

$$
\beta(f)_{-1}[M] \subseteq L \quad \text { and } \quad \beta(f)[\beta L \backslash L] \subseteq \beta M \backslash M .
$$

This is just a general question about Galois adjunctions on coframes: 
In a Galois adjunction

$$
(Y, \leqslant) \underset{\psi}{\longleftarrow}(X, \leqslant)
$$

with $\psi$ a coframe homomorphism (preserving complements) and $\varphi$ its colocalic left adjoint, is there any relation between conditions $\psi(x) \leqslant y$ and $\varphi\left(y^{\prime}\right) \leqslant x^{\prime}$ ?

Fact 8.2. For every $x \in X$ and $y \in Y$,

$$
\psi(x) \leqslant y \Rightarrow \varphi\left(y^{\prime}\right) \leqslant x^{\prime} .
$$

Proof. $\psi(x) \leqslant y \Rightarrow y^{\prime} \leqslant \psi(x)^{\prime}$ and therefore

$$
\varphi\left(y^{\prime}\right) \leqslant \varphi\left(\psi(x)^{\prime}\right)=\varphi(1 \backslash \psi(x))=\varphi(1) \backslash x \leqslant 1 \backslash x=x^{\prime} .
$$

(Evidently, Proposition 8.1 is a particular case of this fact.)

The converse to $(8.1)$ holds whenever $\psi\left(x^{\prime}\right)^{\prime}=\psi(x)$ (note that $\psi\left(x^{\prime}\right)^{\prime} \leqslant \psi(x)$ is always true). Indeed,

$$
\varphi\left(y^{\prime}\right) \leqslant x^{\prime} \Leftrightarrow y^{\prime} \leqslant \psi\left(x^{\prime}\right) \Rightarrow y \geqslant y^{\prime \prime} \geqslant \psi\left(x^{\prime}\right)^{\prime} \geqslant \psi\left(x^{\prime}\right)^{\prime}=\psi(x) .
$$

In particular, this implies that the converse to (8.1) holds whenever $x$ is complemented. Hence:

Fact 8.3. If $X$ is Boolean then the converse to (8.1) holds.

Note that, since $\psi$ preserves complements, then $\psi$ also preserves pseudodifferences in case $X$ is Boolean. Furthermore:

Fact 8.4. If the converse to (8.1) holds, then $\psi$ preserves pseudodifferences.

Proof. Since $\psi\left(x^{\prime}\right) \vee \psi\left(x^{\prime \prime}\right)=1$, we have $\psi\left(x^{\prime}\right) \geqslant \psi\left(x^{\prime \prime}\right)^{\prime}$, that is, $x^{\prime} \geqslant \varphi\left(\psi\left(x^{\prime \prime}\right)^{\prime}\right)$. Then, by hypothesis, it follows that $\psi(x) \leqslant \psi\left(x^{\prime \prime}\right)$ and thus $\psi\left(x^{\prime \prime}\right)=\psi(x)$ (this means in particular that $\psi$ is skeletal). So $\psi\left(x^{\prime \prime}\right)=\psi(x) \geqslant \psi(x)^{\prime \prime}$, that is, $x^{\prime \prime} \geqslant \varphi\left(\psi(x)^{\prime \prime}\right)$ and again by the hypothesis we get $\psi\left(x^{\prime}\right) \leqslant \psi(x)^{\prime}$, which confirms that $\psi\left(x^{\prime}\right)=\psi(x)^{\prime}$ for every $x \in X$.

This shows that in our context, any $\beta$-remainder preserving $f: L \rightarrow M$, with $\beta M$ nonBoolean, such that $\beta(f)_{-1}[-]$ does not preserve pseudodifferences is a counterexample for the converse to Proposition 8.1.

\section{Nearly realcompact frames}

All frames $L$ considered in this section are completely regular.

A Tychonoff space $X$ has been defined by Blair and van Douwen [11] to be nearly realcompact if $X$ is nearly $v X$, that is, $\beta X \backslash v X$ is dense in $\beta X \backslash X$. This was extended to pointfree topology in [18], again via a definition that strongly depends on the points of the frame. Needless to say, we may take a more direct and natural way and just define a completely regular locale $L$ to be nearly realcompact if $\beta L \backslash v L$ is dense in $\beta L \backslash L$. Clearly, any realcompact frame $L$ is nearly realcompact since $v L=L$. 
Remarks 9.1. (a) As in spaces, pseudocompact frames are the frames in which every real-valued function is bounded ([6]). They are also characterized as the frames $L$ for which $v L=\beta L$. Hence, any pseudocompact nearly realcompact frame is compact: $0=\beta L \backslash v L$ dense in $\beta L \backslash L$ implies $\beta L \backslash L=0$. This extends Corollary 4 of [7].

(b) A space $X$ is nearly pseudocompact ([22]) if $v X \backslash X$ is dense in $\beta X \backslash X$. Similarly, let us define a frame $L$ as nearly pseudocompact whenever $v L \backslash L$ is dense in $\beta L \backslash$ $L$. Clearly every pseudocompact frame is nearly pseudocompact and every realcompact nearly pseudocompact frame is compact (since, in that case, $\beta L \backslash L=\overline{v L \backslash L}=0$ ).

The treatment of nearly realcompact frames in [18] is based on a result about dense subspaces (Lemma 3.1) that one can immediately extend to frames with the help of the following lemma:

Lemma 9.2. A sublocale $S$ of a locale $L$ is dense if and only if $S$ meets every nonempty open sublocale of $L$.

Proof. $\Rightarrow$ : Let $U=\mathfrak{o}(a)=\{a \rightarrow x \mid x \in L\}$ be a nonempty open sublocale of $L$. Since $a \neq 0$, then $a^{*} \neq 1$ and, of course, $a^{*}=a \rightarrow 0 \in S$ since $0 \in S$. Hence $1 \neq a^{*} \in S \cap U$, which shows that $S \cap U \neq\{1\}=0$.

$\Leftarrow$ : The case $\mathfrak{c}(a) \supseteq S$ for some $\mathfrak{c}(a) \neq 1$ (i.e., $\mathfrak{o}(a) \neq 0$ ) would imply $\mathfrak{o}(a) \cap S=0$ (because $\mathfrak{o}(a) \cap S \subseteq \mathfrak{o}(a) \cap \mathfrak{c}(a)=0$ ), a contradiction.

Proposition 9.3. Let $L$ be a locale, and $A \subseteq B$ sublocales of $L$. Then $B^{\prime}$ is dense in $A^{\prime}$ if and only if every open sublocale in $L$ which meets $A^{\prime}$ also meets $B^{\prime}$.

Proof. $\Rightarrow$ : Suppose $B^{\prime}$ is dense in $A^{\prime}$ and let $U$ be an open sublocale of $L$ which meets $A^{\prime}$, that is, $U \cap A^{\prime} \neq 0$. Then $U \cap A^{\prime}$ is a nonempty open sublocale of $A^{\prime}$ and, by Lemma 9.2, $B^{\prime} \cap U=B^{\prime} \cap U \cap A^{\prime} \neq 0$.

$\Leftarrow$ : Let $W \neq 0$ be an open sublocale of $A^{\prime}$ and consider an open sublocale $U$ of $L$ such that $W=U \cap A^{\prime}$. By the hypothesis, $U$ meets $B^{\prime}$. Therefore $0 \neq U \cap B^{\prime}=U \cap A^{\prime} \cap B^{\prime}=W \cap B^{\prime}$ and finally, by the Lemma, $B^{\prime}$ is dense in $A^{\prime}$.

Then we get the following characterizations of near realcompactness.

Corollary 9.4. For any completely regular frame $L$, the following conditions are equivalent:

(i) $L$ is nearly realcompact.

(ii) Every open sublocale in $\beta L$ which meets $L^{\prime}$ also meets $(v L)^{\prime}$.

(iii) If there is some $S \in \mathcal{S}(\beta L)$ such that $S \cap L=0$ and $S \cap \mathfrak{o}(a) \neq 0$, then there is some $T \in \mathcal{S}(\beta L)$ such that $T \cap v L=0$ and $T \cap \mathfrak{o}(a) \neq 0$.

Proof. (i) $\Leftrightarrow($ ii) is an immediate consequence of the preceding proposition while (ii) $\Leftrightarrow(i i i)$ follows by using formula (4) of Proposition 4.1 and the fact that any open sublocale distributes over arbitrary joins. 
Hyper-real continuous maps were introduced by Blair in the unpublished manuscript [10]. These are maps that preserve realcompactness by images and pseudocompactness by preimages. Extending Blair's terminology to the pointfree setting, we may say that a localic map $f: L \rightarrow M$ is hyper-real if

$$
\beta(f)[\beta L \backslash v L] \subseteq \beta M \backslash v M .
$$

Lemma 9.5. A frame $L$ is nearly realcompact if and only if

$$
L \vee \operatorname{cl}_{\beta L \backslash L}(\beta L \backslash v L)=\beta L .
$$

Proof. If $L$ is nearly realcompact then $\operatorname{cl}_{\beta L \backslash L}(\beta L \backslash v L)=\beta L \backslash L$. Hence $L \vee \operatorname{cl}_{\beta L \backslash L}(\beta L \backslash$ $v L)=L \vee(\beta L \backslash L)=\beta L$. The converse is also obvious: $L \vee \operatorname{cl}_{\beta L \backslash L}(\beta L \backslash v L)=\beta L$ implies $\operatorname{cl}_{\beta L \backslash L}(\beta L \backslash v L) \supseteq \beta L \backslash L$.

Remark 9.6. As in spaces, given a tower $S \subseteq T \subseteq L$ of sublocales of $L, \operatorname{cl}_{T}(S)=$ $\operatorname{cl}_{L}(S) \cap T$. Hence $\operatorname{cl}_{\beta L \backslash L}(\beta L \backslash v L) \subseteq \operatorname{cl}_{\beta L}(\beta L \backslash v L)$ and it follows from (9.2) that $L \vee \operatorname{cl}_{\beta L}(\beta L \backslash v L)=\beta L$ holds in any nearly realcompact frame.

Lemma 9.7. Let $f: L \rightarrow M$ be a localic map. Then, for every sublocale $S$ of $L$, $f\left[\operatorname{cl}_{L}(S)\right] \subseteq \operatorname{cl}_{M}(f[S])$.

Proof. Actually,

$$
f\left[\operatorname{cl}_{L}(S)\right]=f[\bigcap\{\mathfrak{c}(a) \mid S \subseteq \mathfrak{c}(a)\}]=\bigcap\{f[\mathfrak{c}(a)] \mid S \subseteq \mathfrak{c}(a)\}
$$

and the last intersection is contained in $\operatorname{cl}_{M}(f[S])$ as we now show:

Let $\mathfrak{c}(b)$ with $f[S] \subseteq \mathfrak{c}(b)$. This means that $f(s) \geqslant b$, that is, $s \geqslant f^{*}(b)$, for all $s \in S$. Hence $S \subseteq \mathfrak{c}\left(f^{*}(b)\right)$ and, moreover, $f\left[\mathfrak{c}\left(f^{*}(b)\right)\right] \subseteq \mathfrak{c}(b)$ since $x \geqslant f^{*}(b)$ implies $f(x) \geqslant f f^{*}(b) \geqslant b$.

Lemma 9.8. Let $f: L \rightarrow M$ be an hyper-real localic map. Then

$$
\beta(f)\left[\operatorname{cl}_{\beta L}(\beta L \backslash v L)\right] \subseteq \operatorname{cl}_{\beta M}(\beta M \backslash v M) .
$$

Proof. Apply Lemma 9.7 to get $\beta(f)\left[\operatorname{cl}_{\beta L}(\beta L \backslash v L)\right] \subseteq \mathrm{cl}_{\beta M}(\beta(f)[\beta L \backslash v L])$ and then use condition (9.1) of hyper-real maps.

Finally, we can show that near realcompactness is an invariant property under hyperreal localic maps.

Theorem 9.9. Let $f: L \rightarrow M$ be an hyper-real (localic) map. If $L$ is nearly realcompact and $f$ is a surjection, then $M$ is also nearly realcompact.

Proof. Using Lemma 9.5 we get

$$
M=f[L]=\beta(f)[L] \subseteq \beta(f)[\beta L]=\beta(f)\left[L \vee \operatorname{cl}_{\beta L \backslash L}(\beta L \backslash v L)\right] .
$$

Then, by Remark 9.6, we have

$$
M \subseteq \beta(f)\left[L \vee \operatorname{cl}_{\beta L}(\beta L \backslash v L)\right]=\beta(f)[\beta L] \subseteq \beta M .
$$


In particular, $M$ is dense in $\beta(f)\left[L \vee \operatorname{cl}_{\beta L}(\beta L \backslash v L)\right]$. Since the latter is a compact sublocale of $\beta M$ (because the image of any compact sublocale under a localic map is compact), we may conclude that

$$
\beta(f)\left[L \vee \operatorname{cl}_{\beta L}(\beta L \backslash v L)\right]=\beta M .
$$

Furthermore, by Lemma 9.8, we have

$$
\begin{aligned}
\beta(f)\left[L \vee \operatorname{cl}_{\beta L}(\beta L \backslash v L)\right] & =\beta(f)[L] \vee \beta(f)\left[\operatorname{cl}_{\beta L}(\beta L \backslash v L)\right] \\
& \subseteq M \vee \operatorname{cl}_{\beta M}(\beta M \backslash v M) .
\end{aligned}
$$

Hence $M \vee \operatorname{cl}_{\beta M}(\beta M \backslash v M)=\beta M$, that is, $\operatorname{cl}_{\beta M}(\beta M \backslash v M) \supseteq \beta M \backslash M$, from which it follows that

$$
\operatorname{cl}_{\beta M \backslash M}(\beta M \backslash v M)=\operatorname{cl}_{\beta M}(\beta M \backslash v M) \cap(\beta M \backslash M)=\beta M \backslash M .
$$

This shows that $M$ is nearly realcompact.

Theorem 9.9 is the pointfree version of Theorem 2.8 of [34].

Remark 9.10. If $f: L \rightarrow M$ is an hyper-real map and $M$ is pseudocompact, it is straightforward to check that $L$ is also pseudocompact. Indeed, it follows from $\beta M=v M$ and $\beta(f)[\beta L \backslash v L] \subseteq \beta M \backslash v M$ that $\beta(f)[\beta L \backslash v L]=0$. Then, property $\left(\mathrm{L} 3^{\prime}\right)$ of image maps ensures that $\beta L \backslash v L=0$, that is, $\beta L=v L$.

The results in this section illustrate how remainders in the Stone-Čech compactification may be used in pointfree topology to study special classes of compact-like frames as the near realcompact ones. Much more could be said about e.g. near pseudocompact or nowhere compact frames and their behaviour along localic maps and hyper-real maps but we do not pursue this here, leaving it aside for further investigations.

\section{Comparing our definition with the one of Dube-Naidoo}

In [17] the authors treated the idea of remainder preservation from a different perspective. We conclude this paper with a brief analysis of the relationship between the two approaches. First, let us recall their definition [17, Def. 3.2], here formulated inside the category of locales:

Let $f: L \rightarrow M$ be a localic map between completely regular locales. They say that $f$ takes the remainder of a sublocale $j_{S}: S \rightarrow L$ of $L$ to the remainder of a sublocale $j_{T}: T \rightarrow M$ of $M$ if

$$
j_{T}^{*}(f(p))=1 \text { for every } p \in \Sigma L \text { such that } j_{S}^{*}(p)=1 .
$$

In particular, they say that $f$ is $\beta$-proper (resp. $\lambda$-proper, resp. $v$-proper) if $\beta(f)$ (resp. $\lambda(f)$, resp. $v(f))$ takes the remainder of $L$ to the remainder of $M$.

Remark 10.1. Any $x \in L$ such that $j_{S}^{*}(x)=1$ is necessarily in $L \backslash S$. In fact, $x \in L=$ $S \vee(L \backslash S)$ means that $x=s \wedge r$ for some $s \in S$ and $r \in L \backslash S$; but $j_{S}^{*}(x)=\bigwedge\{s \in S \mid$ $s \geqslant x\}=1$ implies that $s=1$, that is, $x=r \in L \backslash S$. 
For each $a \in L, \mathfrak{b}(a)=\{x \rightarrow a \mid x \in L\}$ is the least sublocale containing $a$ ([29, III.10.2]). In case $a$ is a point $p$ of $L$, we have, for any $x \in L, p=(x \vee p) \wedge(x \rightarrow p)$ and, therefore, $p=x \vee p$ or $p=x \rightarrow p$. Hence

$$
x \rightarrow p= \begin{cases}1 & \text { if } x \leqslant p \\ p & \text { otherwise. }\end{cases}
$$

and $\mathfrak{b}(p)=\{1, p\}$ (these are the one-point sublocales [29]). The case where $p$ is completely prime has a special feature (cf. [4]):

Proposition 10.2. For each $p \in \Sigma L, \mathfrak{b}(p)$ is complemented if and only if $p$ is completely prime.

Proof. $\Rightarrow$ : We have $(L \backslash \mathfrak{b}(p)) \cap \mathfrak{b}(p)=\{1\}$ and hence $p \notin L \backslash \mathfrak{b}(p)$. Since $(L \backslash \mathfrak{b}(p)) \vee \mathfrak{b}(p)=$ $L$, the set $A=\{x \in L \mid x>p\}$ is contained in $L \backslash \mathfrak{b}(p)$ (because no $x>p$ can be obtained as some $y \wedge p$ ) and thus $\bigwedge A \in L \backslash \mathfrak{b}(p)$. Hence $\bigwedge A>p$ which clearly shows that $p$ is completely prime.

$\Leftarrow$ : By Proposition 4.1(4), $L \backslash \mathfrak{b}(p)=\bigvee\{S \in \mathcal{S}(L) \mid S \cap \mathfrak{b}(p)=0\}$. Hence $a \in(L \backslash \mathfrak{b}(p))$ if and only if $a=\bigwedge A$ for some $A \subseteq \bigcup\{S \in \mathcal{S}(L) \mid p \notin S\}$. In particular, $p \notin(L \backslash \mathfrak{b}(p))$ by the complete primeness of $p$. Hence $\mathfrak{b}(p) \cap(L \backslash \mathfrak{b}(p))=0$ and $\mathfrak{b}(p)$ is complemented.

It follows immediately from this proposition that, for any completely prime $p \in L$ and any $S \in \mathcal{S}(L)$,

$$
p \notin S \Leftrightarrow \mathfrak{b}(p) \cap S=0 \Leftrightarrow \mathfrak{b}(p) \subseteq L \backslash S .
$$

Next, we need to recall that a frame $L$ is regular if and only if

$$
a \nless b \quad \Rightarrow \quad \exists c \in L: a \vee c=1 \text { and } c^{*} \leqslant b
$$

for every $a, b \in L$.

Proposition 10.3. Let $L$ be a regular frame. Then:

(1) Every $p \in \Sigma L$ is completely prime.

(2) For each $p \in \Sigma L$ and $S \in \mathcal{S}(L), j_{S}^{*}(p)=1$ iff $p \notin S$.

Proof. (1) Let $p \neq 1$ be a prime element and $p=\bigwedge S$. If $s \$ p$ for every $s \in S$ then, by regularity, there exists for each $s \in S$ some $c_{s} \in L$ such that $c_{s} \vee s=1$ and $c_{s}^{*} \$ p$. But then $c_{s} \wedge c_{s}^{*}=0 \leqslant p$ would imply $c_{s} \leqslant p$ (as the other alternative $c_{s}^{*} \leqslant p$ is impossible) and finally we would get $1=c_{s} \vee s \leqslant p \vee s=s$, that is, $p=\bigwedge S=1$.

(2) The implication “ $\Rightarrow$ ” is obvious since $p \neq 1$. Conversely, if $p \notin S$ then $j_{S}^{*}(p) \$ p$ and thus, by regularity, there is some $c$ satisfying $j_{S}^{*}(p) \vee c=1$ and $c^{*} \leqslant p$. But $c \wedge c^{*}=0 \leqslant p$ and $p$ is meet-irreducible so $c \leqslant p$. Hence $1=j_{S}^{*}(p) \vee c \leqslant j_{S}^{*}(p) \vee p=j_{S}^{*}(p)$.

As any localic map sends points to points ([29, II.3.4]), it is fairly clear that this result together with (10.2) asserts that in regular frames condition (10.1) is equivalent to

$$
\mathfrak{b}(p) \subseteq(L \backslash S) \Rightarrow \mathfrak{b}(f(p)) \subseteq(M \backslash T) .
$$

Hence, we have: 
Corollary 10.4. Let $f: L \rightarrow M$ be a localic map, with $L$ and $M$ regular frames, and let $S \in \mathcal{S}(L)$ and $T \in \mathcal{S}(M)$. If $f$ takes $S$-remainder to $T$-remainder then it satisfies (10.1).

Proof. From $\mathfrak{b}(p) \subseteq L \backslash S$ it follows that $\mathfrak{b}(f(p))=f[\mathfrak{b}(p)] \subseteq f[L \backslash S] \subseteq M \backslash T$, whence (10.3) holds.

Thus for regular frames we have the following picture depicting the relations between the several mentioned classes of localic maps (with none of the indicated implications reversible):

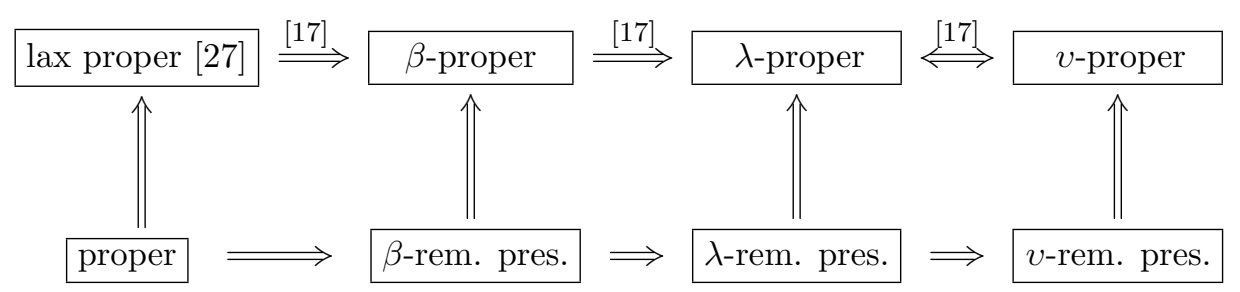

Finally, in order to get the converse to Corollary 10.4 we need to impose some spatiality condition on frames $L$ and $M$, namely the $T_{D}$-axiom, the usual requirement under which topological properties are faithfully described by the pointfree setting. For this, recall that a space $X$ is $T_{D}$ if for each $x \in X$ there is an open $U \ni x$ such that $U \backslash\{x\}$ is still open (clearly, $T_{D}$ is strictly stronger than $T_{0}$ and strictly weaker than $T_{1}$ ). A frame $L$ is $T_{D}$-spatial $([9,30])$ if $L \cong \mathfrak{O} X$ for some $T_{D^{-}}$-space $X$. In the following, $\mathrm{CP}(L)$ will be the set of all completely prime elements of $L$. By [4, Cor. 2.5.2], a frame $L$ is $T_{D}$-spatial if and only if

$$
L=\bigvee\{\mathfrak{b}(p) \mid p \in \mathrm{CP}(L)\}
$$

We have then:

Proposition 10.5. Let $f: L \rightarrow M$ be a localic map, with $L$ and $M$ both $T_{D}$-spatial and regular, and let $S \in \mathcal{S}(L)$ and $T \in \mathcal{S}(M)$. If $f$ satisfies (10.1) then it takes $S$-remainder to $T$-remainder.

Proof. As a consequence of (10.4) and (3.3) we have

$$
L \backslash S=\bigvee_{p \in \mathrm{CP}(L)}(\mathfrak{b}(p) \backslash S)=\bigvee_{p \in \mathrm{CP}(L)}(\mathfrak{b}(p) \cap(L \backslash S))
$$

Therefore

$$
f[L \backslash S]=\bigvee\{f[\mathfrak{b}(p)] \mid p \in \mathrm{CP}(L) \cap(L \backslash S)\}=\bigvee\{\mathfrak{b}(f(p)) \mid p \in \Sigma L, p \notin S\} .
$$

Similarly,

$$
M \backslash T=\bigvee_{q \in \operatorname{CP}(M)}(\mathfrak{b}(q) \cap(M \backslash T))=\bigvee\{\mathfrak{b}(q) \mid q \in \Sigma M, q \notin T\}
$$

Since $p \in \Sigma L$ implies $f(p) \in \Sigma M$ and, by hypothesis, $p \notin S$ implies $f(p) \notin T$, we have $f[L \backslash S] \subseteq M \backslash T$, as required. 


\section{Acknowledgements}

We thank the referee for the careful review and helpful suggestions about exposition.

Research partially supported by the Centre for Mathematics of the University of Coimbra funded by the Portuguese Government through FCT (UID/MAT/00324/2013) and co-funded by the European Regional Development Fund through the Partnership Agreement PT2020. The second named author also acknowledges support from grants MTM2015-63608-P (MINECO/FEDER,UE) and IT974-16 (Basque Government).

\section{References}

[1] D. Baboolal, Perfect compactifications of frames, Czech. Math. J. 61 (2011) 845-861.

[2] D. Baboolal, Conditions under which the least compactification of a regular continuous frame is perfect, Czech. Math. J. 62 (2012) 505-515.

[3] D. Baboolal, $N$-star compactifications of frames, Topology Appl. 168 (2014) 8-15.

[4] D. Baboolal, P. Pillay and A. Pultr, C-connected frame congruences, Categ. Gen. Algebr. Struct. Appl. 6 (2017) 51-66.

[5] B. Banaschewski, Compactification of frames, Math. Nachr. 149 (1990) 105-115.

[6] B. Banaschewski and C. Gilmour, Pseudocompactness and the cozero part of a frame, Comment. Math. Univ. Carolin. 37 (1996) 577-587.

[7] B. Banaschewski and C. Gilmour, Realcompactness and the cozero part of a frame, Appl. Categ. Structures 9 (2001) 395-417.

[8] B. Banaschewski and C. Mulvey, Stone-Čech compactification of locales. I, Houston J. Math. 6 (1980) 301-312.

[9] B. Banaschewski and A. Pultr, Pointfree aspects of the $T_{D}$ axiom of classical topology, Quaest. Math. 33 (2010) 369-385.

[10] R. L. Blair, Mappings that preserve realcompactness, available online at http://at.yorku.ca/t/o/p/d/43.pdf.

[11] R. L. Blair, E. K. van Douwen, Nearly realcompact spaces, Topology Appl. 47 (1992) 209-221.

[12] X. Chen, Closed frame homomorphisms, PhD Thesis, McMaster University, 1991.

[13] X. Chen, Stably closed frame homomorphisms, Cah. Top. Géom. Diff. Catég. 37 (1996) 123-144.

[14] M. M. Clementino, E. Giuli and W. Tholen, A functional approach to general topology, in: Categorical foundations, pp. 103163, Encyclopedia Math. Appl., 97, Cambridge Univ. Press, 2004.

[15] M. M. Clementino, J. Picado and A. Pultr, The other closure and complete sublocales, Appl. Categ. Structures, to appear.

[16] T. Dube, Characterizing realcompact locales via remainders, Georgian Math. J., to appear.

[17] T. Dube and I. Naidoo, A pointfree version of remainder preservation, Categ. Gen. Algebr. Struct. Appl. 1 (2013) 27-58.

[18] T. Dube, I. Naidoo and C. N. Ncube, Nearly realcompact frames, Topology Appl. 168 (2014) 25-39.

[19] R. Engelking, General Topology, Heldermann Verlag, Berlin, 1989.

[20] W. He and M. Luo, A note on proper maps of locales, Appl. Categ. Structures 19 (2011) 505-510.

[21] M. Henriksen and J. R. Isbell, Some properties of compactifications, Duke Math. J. 25 (1958) 83106.

[22] M. Henriksen and M. C. Rayburn, On nearly pseudocompact spaces, Topology Appl. 11 (1980) $161-172$.

[23] J. R. Isbell, First steps in descriptive theory of locales, Trans. Amer. Math. Soc. 327 (1991) 353-371.

[24] P. T. Johnstone, The Gleason cover of a topos, II, J. Pure Appl. Algebra 22 (1981) 229-247.

[25] P. T. Johnstone, Stone Spaces, Cambridge Univ. Press, Cambridge, 1982.

[26] P. T. Johnstone, On solubility of sublocales, Topology Proc. 29 (2005) 527-537.

[27] M. Korostenski and C. C. A. Labuschagne, Lax proper maps of locales J. Pure Appl. Algebra 208 (2007) 655-664.

[28] J. Madden and J. Vermeer, Lindelöf locales and realcompactness, Math. Proc. Camb. Phil. Soc. 99 (1986) 473-480.

[29] J. Picado and A. Pultr, Frames and Locales: topology without points, Frontiers in Mathematics, Vol. 28, Springer, Basel, 2012. 
[30] J. Picado and A. Pultr, New aspects of subfitness in frames and spaces, Appl. Categ. Structures 24 (2016) 703-714.

[31] J. Picado, A. Pultr and A. Tozzi, Joins of closed sublocales, Houston J. Math., to appear.

[32] T. Plewe, Quotient maps of locales, Appl. Categ. Structures 8 (2000) 17-44.

[33] T. Plewe, Sublocale lattices, J. Pure Appl. Algebra 168 (2002) 309-326.

[34] J. J. Schommer and B. Mitra, Maps and f-normal spaces, Topology Appl. 153 (2006) 2735-2744.

[35] W. Tholen, A categorical guide to separation, compactness and perfectness, Homology Homotopy Appl. 1 (1999) 147-161.

[36] J. J. C. Vermeulen, Proper maps of locales, J. Pure Appl. Algebra 92 (1994) 79-107. 